\title{
COBRAR LA PALABRA A LA MUERTE. COMENTARIOS A LA MUERTE DE VIRGILIO DE HERMANN BROCH DESDE LA PERSPECTIVA DE LA TEORÍA PSICOANALÍTICA DE LACAN
}

\author{
Wolfram Bergande \\ Hochschule für Künste \\ Bremen, Alemania \\ wbergande@hfk-bremen.de
}

RESUMEN / ABSTRACT

Hermann Broch y Jacques Lacan comparten -independientemente- el interés por una nueva lógica 'lírica' respectivamente 'creativa'. Broch la esquematiza en sus escritos teóricos y sus cartas y la pone en obra en La muerte de Virgilio. El 'pensamiento paralógico', formulado por el psiquiatra alemán E. v. Domarus, en la psicosis esquizofrénica ayuda a definir los teoremas lógicos de Broch y Lacan. Con tal de arraigar su 'lógica lírica' en una visión-demundo, Broch escogió un paradigma cristiano, a diferencia de Lacan, que siguió a Freud en su rechazo de mitos religiosos.

Palabras Clave: Broch, Lacan, lógica, psicosis, religión.

\section{DEATH, LITERALLY. SOME REMARKS ON HERMANN BROCH'S THE DEATH OF VIRGIL FROM A LACANIAN POINT OF VIEW}

Independently from each other, both Broch and Lacan share an interest in developing a new kind of , lyrical 'resp. , creative ' logic. Broch drafts his new logic in his theoretical writings and his letters and realizes it in his The death of Virgil. The so-called von Domarus principle of ,paralogical thought ' in schizophrenic psychosis can help to define Broch's as well as Lacan's ideas about logic. To frame his, lyrical logic', Broch makes use of a Christian paradigm, whereas Lacan follows Freud in rejecting religious myths.

KEYWORDS: Broch, Lacan, logic, psychosis, religion. 
¿Acaso en una relación política, el lazo que mantenía unidos a los Estados de manera invisible y espiritual, no se disolvió, en la religión, el poder que sujetaba lo invisible, no fue debilitado y aniquilado, acaso los políticos y clérigos no tienen en común que,

como antaño los auguros, no pueden mirarse sin una sonrisa? Kierkegaard, O lo uno o lo otro.

¿Para qué leer La muerte de Virgilio de Hermann Broch? ${ }^{1}$ Considerando las numerosas críticas tanto de la forma como del contenido que la novela de Broch ha sufrido desde su publicación en 1945, esta pregunta se plantea hoy en día no solo a los expertos en Broch (Lützeler 11ff.). El texto presente ni quiere responder de manera concluyente esta pregunta ni resumir todas las respuestas de la literatura secundaria a estas alturas bastante extensa, sino explorar algunos pocos paralelismos que resultan entre la obra literaria, respectivamente, de la obra teórica de Broch, así como algunas de sus cartas, por un lado, y la teoría psicoanalítica de Jacques Lacan, por el otro.

La relación de Broch con el psicoanálisis en general ya ha sido tematizada (por ejemplo, Mondon 516f.). Broch primero fue analizando de "la alumna de Freud, Hedwig Schaxel-Hoffer" (Broch, Broch y Lützeler 15), luego, a partir de 1943 de Paul Federn (Lützeler, Moderne 147-68); su hijo, "Herrmann Friedrich Maria" (Lützeler, Biographie 386) estuvo en tratamiento durante un periodo breve con Rudolph Loewenstein, el analista de Lacan (Broch, Broch y Lützeler 15f.). Aparte de la psicología del yo de su analista Federn, con el que compartió un interés por lo que es del orden del proceso primario en el sueño y en la psicosis (Federn, Ich-Psychologie), Broch, entre otros, fue influido por la psicología analítica de Carl Gustav Jung (Ritzer 535f.; Martens 68); no por último, Broch se manifestó teóricamente respecto del psicoanálisis (Broch, "Psychoanalyse").

Asimismo, la literatura secundaria ya ha considerado una serie de posibles paralelismos entre Broch y el psicoanálisis de Lacan (Mondon 512f.; Edelmann 174; Broch, Lützeler y Federn 17). De los textos de Broch, es especialmente La muerte de Virgilio el que se ofrece para una lectura psicoanalítica, ya que ha sido considerado, como sublimación literaria a gran escala de la

En tiempos de Broch, esta pregunta se planteaba con miras a la Eneida de Virgilio; "Wer liest heute [1926] Vergil?" (Vergilius Maro, Holzberg y Schauer 29), antes de que en los años 1930 en Alemania se produjera cierto renacimiento de Virgilio. 
“angustia intrapsíquica de destrucción” (Dahl 122). Desde el punto de vista contemporáneo, sin embargo, el texto es particularmente interesante, porque, como sobre todo Edelmann argumenta de manera convincente, en algunos de sus pasajes aparece marcado por el "autoconocimiento psicoanalítico" (Edelmann 106; cf. Gabbiadini 215f.) de su autor; de este modo, la novela puede ser interpretada como una suerte de autoterapia con medios literarios, como "terapia literaria en contra de su voluntad" (Edelmann 89ff.), a saber, una terapia que se remite a los elementos en común "entre el sueño y el arte, sobre todo entre el sueño y la lírica" (Broch y Lützeler, $K W$ 13, 2 359) así como entre "poética" y lógica del sueño (Edelmann 152). Ante este trasfondo, la expectativa de que en la obra de Broch se hallen introspecciones literarias o teóricas que también resulten relevantes para la teoría psicoanalítica contemporánea, es más que fundada.

\section{LAANGUSTIA DE LA SOCIEDAD SIN PADRE(S)}

Desde un punto de vista filosófico o metapsicológico, Broch y Lacan comparten la afinidad a una comprensión kierkegaardiana del sujeto: el sujeto moderno, ilustrado no es que realmente se asiente en la razón, sino que naufraga en la época que Hegel definió como lo romántico cristiano, en una posición de la angustia, del aislamiento y del estar escindido. Dado que vive en el umbral hacia el cristianismo, durante el principado del emperador romano Octaviano ('Augustus'), también para el protagonista Virgilio en La muerte de Virgilio así como en La fenomenología del espíritu de Hegel, las antiguas ideologías de esclavos (estoicismo, escepticismo) desembocan en la conciencia desdichada, es decir, en una autoconciencia que intenta recuperar su ser que desaparece en un "más allá inalcanzable" (Hegel 144).

"[...] esa conciencia pone y mantiene juntos el puro pensamiento $y$ la individualidad [la Einzelnheit], pero todavía no se ha levantado [es decir, no se ha elevado] a aquel pensamiento para el que la individualidad o particularidad de la conciencia [es decir, la particularidad de la conciencia, esto es, el quedar esta suelta y perdida ahí, la Einzelnheit de la conciencia] ha hecho las paces con el pensamiento puro mismo. Esa conciencia se encuentra más bien en ese punto medio en el que el pensamiento abstracto toca la individualidad de la conciencia como individualidad [su Einzelnheit como Einzelnheit]. Ella misma es ese contacto; ella es la unidad del puro pensamiento y la individualidad 
[Einzelnheit] [la unidad del pensamiento puro y el quedar ahí suelta la conciencia]; [...] Por tanto, [...] la conciencia se comporta respecto a su objeto no pensantemente [en el sentido de lo que hemos llamado pensamiento], sino en cuanto ella misma, ciertamente, es en sí pura individualidad pensante y su objeto es también exactamente ése o eso [es decir, su objeto es también esa pura individualidad pensante], pero la relación del uno con el otro y del otro con el uno no es todavía ella misma puro pensamiento, la conciencia no hace (por decirlo así) sino enderezarse al pensamiento [Denken], tensarse en aspiración a él, y es Andacht [es decir, suspirante attentio, religio y pietas], devoción" (Hegel 143/320-321).

El individuo hace de sí la experiencia como 'pura individualidad pensante', pero, de manera semejante al soñante o al psicótico, en este pensamiento no es subjetivado propiamente tal, no es verdaderamente $\operatorname{cosido}^{2}$ o "anclado" (Federn, Zur Psychologie der Revolution 28f.), sino que piensa y pasa de largo de su propio pensamiento, tan solo lo toca. Este tocar o comenzar a pensar, respectivamente, pensar rememorante [An-Denken] es expresión sintomática del hecho de que no se comprende respectivamente no puede comprenderse como aquel que ex-siste, él mismo, como la 'relación' viva, que necesariamente ha de ser presupuestada, entre singularidad o individualidad [Einzelheit] y pensamiento. Así busca, al igual que el Virgilio del Virgilio, esta relación y, con eso, se busca a sí mismo, es decir, su sujeto del inconsciente, en un más allá del pensamiento, respectivamente, "más allá del lenguaje" (Broch, Vergil $454)^{3}$ y delega la mediación añorada a otro absoluto -en el caso de Virgilio, al redentor cristiano 4 respectivamente a un "arquetipo" jungiano "(a saber, el del niño divino)" (Waldeck 127).

Su pensamiento como tal se queda en ese zumbido sin forma que deja el repique de las campanas o en un henchirse de cálida niebla, se queda en un pensamiento musical que no llega al concepto, concepto que aquí [en esta forma de la conciencia que estamos considerando] sería la única forma o manera objetual inmanente [es decir, que aquí sería la única forma y manera que inmanentemente podría tener ella el

\footnotetext{
'Cosido' o 'pespunteado' en el sentido del teorema lacaniano del point de capiton.

$C f$. Lawrence sobre el Virgilio: "Thought ultimately transcends linguistic expression" (Lawrence 320).

$4 \quad$ En definitiva, para la superación de la muerte ( $c f$. Roethke 147).
} 
carácter de objeto]. A este infinito y puro sentimiento interno le nace, ciertamente, un objeto; pero es un objeto que se presenta de suerte que no lo hace como entendido [como begriffen, como concepto] [es decir, que no se presenta como lo otro que resulta no ser sino la conciencia misma] y que, por tanto, se presenta como algo extraño (Hegel 143).

El sentimiento meramente piadoso, contingente y la extrañeza no comprendida que Hegel diagnostica, caracterizan también al monólogo interior del protagonista de Broch que es Virgilio. En el Virgilio, así como también en muchos de los demás escritos teóricos de Broch, este momento del serextraño y de la falta de sostén siempre remiten también a un déficit social. Con esto, Broch sigue escribiendo un diagnóstico que también es discutido en Federn (La sociedad sin padres), Freud y después en A. Mitscherlich (En camino hacia una sociedad sin padres) y también en Lacan (por ejemplo, a propósito de la interpretación de Le père humilié de Paul Claudel) ${ }^{5}$ : las instancias constituyentes de unidad, que abren el camino para la socialización del individuo y de su socialización, colapsan con la Modernidad.

A excepción de las ciencias, de acuerdo a la Estética de Hegel, son las artes más avanzadas, al ser las más fuertemente reflexionadas subjetivamente, tales como el humor de la comedia antigua o la ironía del Romanticismo (por ejemplo, Nubes de Aristófanes o Rede des toten Christus vom Weltgebäude herab, dass kein Gott sei [Discurso del Cristo muerto desde lo alto del edificio del mundo de que no exista Dios] de Jean Paul), las que disuelven estos garantes metafísicos; esto vale, en la misma medida, para la Antigüedad greco-romana así como para la época 'romántica' del cristianismo latino cuyo final Hegel escribía -y Broch padecía. En este sentido, el Virgilio de

Federn (1919 28f.) y Freud (1930) discuten esta pregunta en el ejemplo de la sociedad norteamericana de esa época. Federn depositaba su esperanza en una hermandad de los emigrantes que pudieron dejar sus relaciones paternas patógenas en el viejo continente. Freud, en cambio, diagnosticaba en la sociedad norteamericana contemporánea "la miseria psicológica de la masa" (Freud, Unbehagen 87), que es creado ahí "donde la ligazón social principalmente es producida por identificación de los participantes entre ellos, mientras que individualidades líderes no adquieren la relevancia que les debía ser otorgada en la formación de masas" (Freud, Unbehagen 87f.). Como es consabido, Freud era contemporáneo de Mussolini, Hitler y Stalin; es decir, quizá podría haber anticipado en sueños la medida en qué dicha 'miseria' puede ser instrumentalizada políticamente (últimamente durante la presidencia de EE.UU. de Donald Trump). 
Broch explica, frente a Augustus: "Precisamente el gran arte, el que sabe de su misión cognitiva, sabe también de la pérdida del conocimiento y de la pérdida del dios, que hemos sufrido; [...]" (Broch, Vergil 322; $c f$. Heizmann, Antike 177). Para la Modernidad, Dios no solo ha sido extraviado, sino que está muerto (Nietzsche), de manera que la religión le da la espalda, cada vez más, al discurso artístico y científico: hacia la obra de arte total, el protestantismo cultural o el interior de una espiritualidad difusa, pero aparentemente indisoluble. Es por esto que surge la pregunta de si la muerte de Dios bajo la forma religiosa de un sentimiento de culpa internalizado puede seguir constituyendo la moral de la sociedad ilustrada-revolucionaria de la hermandad -sobre todo ante el trasfondo de la incipiente introspección de que Dios no solo está perdido o muerto (porque su muerte incluso es parte del dogma cristiano), sino que desde siempre ha estado muerto ${ }^{6}$, es decir, nunca ha sido más que una mera referencia mítica. Y, en caso de que los formatos religiosos de la muerte de Dios ya no pueden cumplir lo anterior, entonces, ¿quizá sí las ficciones literarias puedan responder a ello? En caso de que el Virgilio de Broch tuviera esta tarea, entonces, al igual que el Wagner tardío, presumiblemente solo embalsamaría la religión en arte $^{7}$.

Ante el trasfondo de un lazo social que en la longe durée se va disolviendo cada vez más y de una identidad individual que se diluye cada vez más, tanto Broch como Lacan adhieren -aunque de manera diferente- a una referencia a una 'unidad', respectivamente, a un 'Uno' -una referencia que si bien tan solo puede ser formulada ficcionalmente (mítica o bien literariamente). Broch se orienta según un paradigma religioso (cristiano) del redentor, por ejemplo, cuando en su teoría del delirio de masas les atribuye a los hombres la culpa del fin de una época dorada pasada y quiere reconocer el colapso de una unidad absoluta como momento histórico que retorna:

6 En este sentido, Guattari cuestiona la referencia absoluta internalizada bajo la forma del superyó respectivamente del ideal del yo y exige "[...] la afirmación de la finitud de toda empresa humana, la falta de toda garantía trascendental, la muerte de Dios en lugar de la muerte del padre que despierta sentimientos de culpa y de sus amenazas de castración en la iniciación edípica" (Guattari 89).

Lützeler señala que Broch consideró al Virgilio también como contribución "mística" (Lützeler, Biographie 301) a la reeducación de los alemanes después de la Segunda Guerra Mundial. 
El terror que asaltó a Virgilio ante el nuevo hombre romano de masas era el mismo al que se vieron expuestos Rousseau y Tolstoi en los periodos de transformación del siglo XVIII y XIX, y tanto Virgilio como Rousseau (y, con un giro hacia lo cristiano primordial, Tolstoi) se convirtieron en proclamadores de una vida orientada hacia la naturaleza, cercana a la naturaleza y dada por la misma, en cuya imagen resplandece, de manera transfigurada, el sueño de la Época Dorada, el sueño de la perfección de la dicha insuperada, insuperable, de antaño, que el hombre por su propia culpa no pudo retener, que por su propia culpa disminuyó más y más (Broch y Lützeler, Massenwahn 144).

Broch reconoce en la religión potenciales para el desarrollo de la humanidad, por ejemplo, la capacidad de despertar al hombre de su "estado crepuscular" (Broch y Lützeler, Massenwahn 181ff.; 154; 71). Frente a esto, Lacan, con Freud, señala a la neurosis obsesiva como eventual variación religiosa de ese Uno (Lacan, XIII 01.12.1965) y le atestigua a la revelación cristiana "una denegación de la verdad como causa, a saber, que ella deniega aquello que funda al sujeto, al tenerse por una parte integral de ello" (Lacan, XIII 01.12.1965). Y, en oposición diametral a Broch, Lacan denuncia el sentimiento de culpa resultante como algo, en última instancia, no científico:

Digamos que el religioso deja a Dios a cargo de la causa, pero que en eso corta su propio acceso a la verdad. Esto también lo lleva a transferir a Dios la causa de su deseo, lo que es propiamente tal el objeto del sacrificio. Su deseo está sometido al supuesto deseo de un dios que por ende hay que seducir. El juego del amor comienza ahí. El religioso instala de esta manera la verdad en un estado de culpabilidad (Lacan, XIII 01.12.1965).

\section{LA LÓGICA LÍRICA EN LA MUERTE DE VIRGILIO}

Es interesante que Broch intente reconstruir esta instancia constituyente de unidad ("unidad como valor fundamental" (Broch, $K W 10 / 2$ 161)) ${ }^{8}$ mediante

\footnotetext{
"Es la unidad del hombre, sostenido por el carácter absoluto del logos, la que se ilumina en la unidad de cada cosa" (Broch, $K W$ 10/2 162).
} 
una forma especial de lógica ${ }^{9}$-de manera parecida a Lacan, que en sus seminarios XV L'acte psychanalytique y XVI D'un Autre à l'autre construye esta instancia constituyente de unidad con los conceptos de una lógica aristotélica ampliada y de esta manera crea uno de sus 'matemas' centrales: el padre mítico de la horda primordial de Tótem y tabú de Freud es el Uno que, en tanto un más allá trascendental (de la ley, del lenguaje), siempre ya tiene que presuponerse. Para la comprensión, de parte de Lacan, de la subjetividad tanto individual como colectiva, este "Uno" (Lacan, Sinthome 13.04.1974) es necesario en la medida en que constituye la identidad autoconsciente del clan de los hermanos mediante una 'lógica de la excepción constitutiva'. De acuerdo a Lacan, incluso fundamenta la categoría de la necesidad en cuanto tal, incluso cuando, desde un punto de vista metapsicológico, pone "en apuros" al psicoanalista (Lacan, Sinthome 13.04.1976). El "Uno" en Lacan, más que una ficción literaria, es una ficción jurídica: el Uno que necesariamente hay que presuponer es el last man standing de cara a la muerte que es inminente como consecuencia de la guerra social de todos contra todos. El padre, respectivamente, las instancias subjetivantes que lo reemplazan, para Lacan son tan solo el mal menor (père ou pire) de cara a las psicosis o de una revolución histérica que, sin saberlo, demanda un -nuevo- padre ('Quieren un amo, lo obtendrán', así Lacan en las escaleras del panteón parisino a los estudiantes en plena revuelta del 68).

Broch principalmente se aproxima a la lógica de la unidad, respectivamente, del Uno trascendental, en dos arranques: primero, cuando en Sobre unidades sintácticas y cognitivas (1946) esboza una "lógica onírica infinitamentedimensional" que iría más allá de la interpretación de los sueños freudiana, en la medida en que "aprehende la totalidad de la variedad relacional infinitamente-dimensional [de un sujeto]" (Broch, $K W 10 / 2$ 262), y que puso a prueba en su Teoría del delirio en masas en un sueño propio (de dos "golpes de campana") (Broch y Lützeler, Massenwahn 439ff.; cf. Edelmann, 145f.) ${ }^{10}$.

9 De manera parecida al Lacan tardío, Broch, al menos de manera preliminar, persigue la idea de representar esta lógica "topológicamente" (Broch y Lützeler, $K W$ 13,2 451) (así Broch en una carta a Aldous Huxley 1945), es decir, mediante la topología matemática.

10 A todo esto, también Jung, en 1934, se había lamentado de la complejidad "inauditamente múltiple, variable y casi incalculable" de la "técnica" psicoanalítica y había vuelto el carácter condicionado por la cosmovisión, relacionado con lo anterior, en contra de "la predisposición materialista [freudiana] en relación a la función religiosa del alma" (Jung 7) - en lugar de tomar esta introspección como motivo para interrogar los propios supuestos metafísicos. 
Segundo, cuando con y en La muerte de Virgilio dice haber 'detectado', según consta en una carta a Hans Sahl del 11.11.1943, una particular "lógica lírica" (Broch y Lützeler, $K W 13,2$ 359; $c f$. Jenkins 194f.); una lógica que, según él, sería capaz de aunar todas las contradicciones de la existencia, según expone también en una carta (29.7.1945) a Federn:

Más bien, Usted [Paul Federn] -ojalá- aún descubrirá que para mí siempre se trató de desocultar lo contradictorio en todo nuestro pensar $\mathrm{y}$ actuar, esa simultaneidad de negro y blanco que precisamente al analista le es más conocida que a cualquier otro, y de demostrar cómo, a pesar de toda contradictoriedad, la unidad del alma, el continuo interior, reflejado en el yo, en general se mantiene. De esto resulta de manera verdaderamente anatómica mi método de representación, a saber, una lírica. Porque el poema lírico desocultó aquella esfera del alma, en la que se integran sus contradicciones. El todo [es decir La muerte de Virgilio] conforma, entonces, un poema lírico y, como todos, es condicionado desde lo musical, obtiene de éste su forma básica. También las oraciones largas [en La muerte de Virgilio] tienen que ser entendidas desde este ángulo; el poema sobredimensionado también exigía elementos sobredimensionados de construcción, tanto más que cada uno de ellos a su vez tenía que obtener todo lo contradictorio junto a su disolución (Broch, Lützeler y Federn 98).

Lacan había introducido la contradicción contradictoria a lo inconsciente que, según Freud, era libre de contradicción y negación, mediante una "lógica elástica" (Lacan, XV 25.01.1968) o más bien mediante una silogística elástica, en la que una oración subcontraria, 'creativamente negada' ( $c f$. Lacan, XV 06.03.1968) media entre dos oraciones contradictorias. Esta negación, en Lacan llamada 'creativa', es una doble negación que, al igual que la suspensión hegeliana, no suspende hacia nada ni hacia la oración de partida, sino que genera algo nuevo. Algo parecido vale para Broch: el "carácter absoluto del logos", que constituye la "unidad del hombre" y "se ilumina en la unidad de cada cosa" no es una tautológica "proyección [...] al vacío" (Broch, $K W$ 10/2 253), según Broch escribe en Sobre unidades sintácticas y cognitivas (cf. Illi 146f.). A saber, porque la presunta tautología del Yo soy yo (Hegel), si se refleja con ayuda de respectivamente en esa instancia absoluta (en dios respectivamente el puro pensamiento (logos)), según Broch no engendra Doppelgänger narcisista alguno (como el moi imaginario en el estadio del espejo de Lacan), sino que genera una dinámica productiva, mediante la que se produce a sí y al yo. El que la tautología no sea hueca, de acuerdo a Broch, 
concretamente tiene que ver con que esa instancia lógica (Dios) "incluso" (Broch, $K W$ 10/2 253) cuando su existencia es negada, lógicamente es productiva - con Lacan habría que agregar: justamente cuando su existencia es negada, es decir: para el hombre moderno de hecho solo cuando su existencia es negada, solo cuando esa existencia es algo como el padre de la horda primordial freudiano, desde siempre ya muerto (matado):

[...] [E]l Dios que es la imagen a semejanza del hombre no es que duplique a éste, sino que es algo diferente, lo es incluso, expresado de manera algo paradojal, ya gracias a su no-existencia, es decir, gracias a las operaciones lógicas a las que les es dado realizar posiciones en lo no-existente. También la ecuación matemática posee carácter tautológico y no solo es una escisión de una identidad, sino que es apertura [Aufschluss] de estructuras lógicas e introspección productiva hacia su interior (Broch, $K W 10 / 2253$ ).

Según muestra la cita anterior, tanto para Broch como para Lacan, la 'unidad' trascendental de la conciencia del yo es función de otro absoluto doblemente negado, a saber, aquí aparentemente de un Dios monoteísta que es no-yo y que, de acuerdo a Broch, 'incluso' (con Lacan: justamente, es decir, solo) en tanto 'no-existente', temporaliza efectos subjetivantes. La oración que formula esa no-existencia del no-yo y que en este lugar podría ser puesto en boca de Broch: 'No hay uno solo que sea no-yo', por lo tanto, al igual que en Lacan ('No hay uno solo que no esté sometido a la castración'; $c f$. Lacan, XV 06.03.1968) la oración mediadora de un "silogismo" (Broch, $K W$ 10/2 165) omniabarcativo en la medida en que conecta a todas las 'unidades cognitivas respectivamente sintácticas' del discurso del sujeto -o bien desde siempre las debe haber unido, desde las sociedades prehistóricas hasta las actuales, según sospecha Broch en Logik einer zerfallenden Welt (1931) ${ }^{11}$. Semejante "posición de la posición" del Otro vía "mundo", y sea, según lo expuesto más arriba, ex negativo, como posición de la no-existencia del Otro,

11 Desde la perspectiva de la "lógica de la investigación" de Karl Popper justamente tales "proposiciones universales del tipo 'hay' [...] no son falsificables: ninguna proposición especial (ninguna proposición de base) puede estar en oposición lógica a la proposición universal 'hay': 'Hay cuervos blancos'. (Solo una proposición universal puede contradecir a semejante proposición). Es por esto que sobre la base de nuestros criterios de delimitación tendremos que designar las proposiciones universales 'hay' como no-empíricas ('metafísicas')" (Popper 46). 
es para Broch la condición de posibilidad para siquiera percibir al 'prójimo' [Nebenmensch], al "yo prójimo" [Neben-Ich] (Broch, KW 10/2 170f.).

En esto, a todo esto, Broch -al igual que Lacan-calificaba las paradojas discutidas por la contemporánea investigación matemático-lógica de los fundamentos, tal como las "antinomias de la infinitud" (Broch, $K W$ 10/2 166) de la teoría matemática de conjuntos como pruebas de la validez de semejante posición, así como de la separación abismal entre subjetividad y mundo que puede ser descrito en términos matemáticamente-positivistas (acaso en el sentido del empirismo lógico del Círculo de Viena, con el que Broch, como es consabido, a pesar de lo anterior mantenía cierta cercanía (cf. Illi 102; $c f$. Roesler-Graichen 53ff.; 147).

Lo productivo propiamente tal que debe partir de esa posición de la no-existencia del Otro ('No hay uno solo que sea no-yo') puede formularse de la siguiente manera: la oración ni se remonta a nada ni a la oración de partida negada, es decir, a 'Hay uno que es no-yo', lo que sería un retroceso a la posición del no-yo (Dios) como ser absoluto (es decir, que significaría o megalomanía o nimiedad de la propia existencia), sino a algo nuevo. 'No hay uno solo que sea no-yo' en ese caso significaría: '(Incluso) todo es no-yo', es decir, también el yo pensante, consciente, pertenece al no-yo. De esto sigue que tiene que haber otro yo, acaso un yo-de-lo-inconsciente, que funda esa 'totalidad del no-yo', a la que justamente también pertenece el yo consciente, pensante - de hecho, uno que precisamente ya no sería transcendente, sino un yo-de-lo-inconsciente en cada caso propio, como cuyo producto se comprende el yo. Entender a este resultado 'productivo' (Broch), respectivamente, 'creativo' (Lacan), de la doble negación -en realidad, entender el resultado de la respectiva adopción de perspectiva entre el yo y el no-yo (después de la muerte de Dios se trata de un proceso estrictamente intrasubjetivo)- pues, entender este resultado significaría consumar el proceso que Hegel en la Fenomenología exigía para la 'piedad subjetiva' (Lasson) de la conciencia infeliz: entender 'el puro sentimiento interior infinito' que, según Hegel, ingresa a la conciencia como algo extraño y efectivamente, tal como ocurre en la psicosis esquizofrénica, de manera regular es experimentado como algo demoníacamente "ajeno" (von Domarus, "Prälogisches" 90), como lo es en cada caso propio.

Si es que falta ese Otro doblemente negado (el 'Uno' como el 'no-yo' absoluto) en el fundamento de aquello que Hegel llama 'el puro sentimiento interior infinito' y Broch el 'continuo interior', entonces, tampoco tiene lugar el yo como sujeto posible de su propia 'variedad infinito-dimensional 
relacional' experimentada (Broch), o bien se convierte, él mismo, en un sujeto absoluto, de manera que entonces, sin embargo, la unidad subjetiva del o de los otros se desintegra de manera discrecional. En cierto modo en paralelo a Broch y a Lacan, el psiquiatra alemán Eilhard von Domarus ${ }^{12}$ y, en su estela, Silvano Arieti en su Interpretation of Schizophrenia (Arieti 229ff.) describen a este proceso con respecto a la esquizofrenia como desintegración de un silogismo: en el "pensamiento paralógico" (von Domarus, "Prälogisches" 87) del esquizofrénico falla la mediación (dialéctica, asilogística, doblemente negante, aristotélica) entre yo y no-yo y es reemplazada por una platónica 'ley de la participación' (von Domarus, "Prälogisches" 87), primero de todo aquello que es no-yo, luego de un yo-absoluto ${ }^{13}$. Hablando lógicamente, en ese caso los predicados ya no pueden ser referidos a este o justamente a otro sujeto, ya que existe un solo sujeto, a saber, el yo, el que, por cierto, es infundado por el no-yo en su sentir y pensar rememorador (An-Denken) infinitos y se hunde en la absoluta identidad con este otro. Dado que ya tan solo hay un sujeto, ya no es posible servirse de la subjetividad como característica distintiva de identidad. Es por eso que ciertos elementos en común, percibidos a voluntad, pueden conducir a identificaciones con los más variados objetos (y en esa medida, del todo en oposición al teorema de la identificación con un 'rasgo unario' que Lacan destaca en Freud). Von Domarus: “[W]hereas the logician accepts identity only upon the basis of identical subjects, the paralogician accepts identity based upon identical predicates" (von Domarus, "Laws" 111). Von Domarus da el siguiente ejemplo:

A schizophrenic patient of the Insane Asylum of the University of Bonn believed that Jesus, cigar boxes, and sex were identical. How

13 Al igual que otros teóricos de los años 1920, von Domarus respecto de esta "ley de la participación' (Lévy-Bruhl 80,378, 454) por lo visto se refiere a Les fonctions mentales dans les sociétés inférieures de L. Lévy-Bruhl: "L'entendement proprement dit tend vers une unité logique, et il en proclame la nécessité. Mais, en fait, notre activité mentale est à la fois rationelle et irrationelle. Le prélogique et le mystique y coexistent avec le logique" (Lévy-Bruhl 455). Sin embargo, la ley de la participación deja interpretarse como methexis genuinamente platónica ( $c f$. acerca del platonismo de Broch: Brinkmann 196), respectivamente en el sentido del teorema positivista contemporáneo del teorema type-token de la llamada filosofía analítica del lenguaje angloamericana. En este sentido, por ejemplo, el debate sobre los actos de habla entre Jacques Derrida y John Searle puede reconducirse a la oposición entre una teoría del signo dialéctica (aristotélica) y otra positivista (platónica). 
did he arrive at that strange belief? Investigation revealed that the missing link for the connection between Jesus, cigar box, and sex was supplied by the idea of being encircled. In the opinion of this patient the head of Jesus, as of a saint, is encircled by a halo, the package of cigars by the tax band, and the woman by the sex glance of the man" (von Domarus, "Laws" 108f.).

De acuerdo al mito cristiano, Jesús sería la mediación absoluta propiamente tal entre Dios (no-yo) y el hombre (yo); una mediación en la que el dios cristiano -como constatan tanto Hegel (en su filosofía de la religión) como Freud (en Tótem y tabú) - se sacrifica (niega) a sí mismo y así produce una identificación silogística (syn-lógica), a saber, justamente al lograr la doble negación del 'Uno’ discutida más arriba en Lacan y Broch. En el caso ejemplar del paciente de Bonn, citado por Domarus, Jesús, en cambio, solo es uno de muchos predicados salvajemente identificados que están referidos a un sujeto indeterminado, a saber, el yo del 'paralógico' inundado por el no-yo ${ }^{14}$.

¿Y qué tiene que ver todo esto con La muerte de Virgilio de Broch? El estilo literario de Broch se convierte en el discurso paralógico del esquizofrénico cuando, en cierto modo, en la experiencia interna de Virgilio suelda o sutura la 'variedad relacional infinito-dimensional' de predicados contradictorios (respectivamente contrarios) (la 'simultaneidad de negro y blanco') mediante ardides gramaticales y, a través de esto, se refiere a un yo absoluto, a saber, el yo endofásico de Virgilio. La endofasia de Virgilio deriva, huye a través de las cadenas de predicados lógicos, que proliferan, alejándose, en todas las direcciones -sin poder estabilizar al propio yo, que es el sujeto lógico de esta experiencia, mediante una referencia identificatoria a un no-yo contrapuesto (a un dios o justamente al propio sujeto del inconsciente), en "a mobile balance that must always be established anew" (Weiss 220).

En su tesis doctoral The logical structure of mind, von Domarus tematiza lo que aquí ha sido llamado el cosido, entrelazamiento o arraigo faltante entre pensar y ser, bajo el recurso a la problematización heideggeriana de la metabasis eis allos genos aristotélica como 'sustancialización' de un predicado en la lógica silogística. De manera parecida a lo realizado por Lacan en su 'lógica del supuesto constitutivo', von Domarus localiza a esta sustancialización en el paso de un enunciado particular respectivamente un enunciado de existencia a un enunciado universal: "Every predication concerning a predicable -and all such syllogisms are cases in point- conceals the thinking in expressing the thought". (von Domarus, The Logical Structure of Mind 50f.). 
En la literatura secundaria sobre el Virgilio de Broch se describen algunos de estos ardides gramaticales. Al igual que Lacan, que también trabajó en ampliar la lógica mediante la gramática ( $c f$. Lacan, XVI 23.04.1969), la gramática de Broch exhibe profundidades del sentido que le escapan a la lógica clásica: "On ne peut pas dire que la langue de Broch soit agrammaticale ou qu'elle transgresse les lois de la grammaire allemande: elle en rélève seulement des possibilités cachées" (Pérennec 164). Broch suelda predicados contradictorios respectivamente contrarios sobre todo mediante un uso sistemático del "oxímoron" (Pérennec 153) o bien mediante "composita" y "ensamblajes" (Lube 128ff.) no habituales en la lengua cotidiana; Lube al respecto ofrece una "Pequeña enciclopedia de las creaciones lingüísticas" en el Virgilio, por ejemplo, "imagen del ruido", "ataque-huida", "polvo plateado del eco", "raudales de piedras", "maraña cantante de formas", "cueva del adentro y afuera", "paisaje del sonido primordial", "claridad nocturna", "noche de luminosidad", "lejano-cercano", "duelo del inespacio", "aroma (a) corriente", "jadeo de piedras", "perder la vista como piedra", "frío como un sueño" (Lube 128 ff.) o "arder gélidamente" (Lube 59). Entre las "infinitas letanías" (Broch y Lützeler, $K W 13,2$ 451), como el propio Broch en una ocasión se refiere a sus construcciones de oraciones en el Virgilio, también se encuentran tautologías que recuerdan la calificación, de parte de Lacan, de algunos elementos del discurso psicótico como "ritournelle" (Lacan, III 44), así como una figura etymologica que por un incremento poco habitual del adjetivo se vuelve aún más tautológica: "más sola soledad" (Pérennec 157).

Dado que a los predicados lógicos, por ejemplo, a los adjetivos, en el discurso interior de Virgilio les falta, de manera fundamental, la doble negación expuesta más arriba, se disuelve su lógica de la lengua cotidiana, $\mathrm{y}$, en tanto gramaticalmente negados de manera simple, ganan una nueva dimensión constituyente de sentido, que a su vez recuerda fuertemente a la discusión de Lacan de la negación y en particular del francés "ne explétif" (Lacan, XV 28.02.1968). Según muestra Pérennec, Broch articula esta dimensión, por ejemplo, al incrementar y negar adjetivos que en realidad no pueden ser incrementados: por ejemplo, "más no bello" [unschöner], "más no inteligente" [unklüger] (Pérennec 157). Esto significa "que la negation ou l'absence d'une qualité chez Broch ne sont pas conçues de manière totalement négative, pusique cette absence est elle-même susceptible d'évolution, de graduation. Le pôle négatif des adjectifs acquiert chez Broch les mêmes vertus dynamiques qu'il n'a pas en langue dite standard" (Pérennec 157). La fusión de predicados lógicamente contrarios o bien contradictorios por lo 
visto tiene el mismo objetivo que el "valeur asymptotique du superlatif" (158) constatado por Pérennec o la suspensión "[de] toute signification temporelle" (Pérennec 159; $c f$. Lube 54) mediante construcciones participiales, a saber, el objetivo de alcanzar una realidad total, respectivamente una totalidad real ${ }^{15}$ : "[T] oute qualité négative comporte en soi l'affirmation de la valeur positive correspondante et balaye [inunda] par l'oxymoron la totalité de la qualité envisagée" (Pérennec 160f.). En esto, para Broch se trata, en última instancia, de "la totalidad indominable del mundo, porque solamente en lo total debería dejarse encontrar un fundamento inmutable de todo saber, si es que no ha de seguir siendo uno indomable“" (Broch y Lützeler 51) ${ }^{16}$.

En cierto sentido Broch incesantemente forma 'palabras primordiales' antitéticas en el sentido de Carl Abel, a quien Freud reiteradamente cita, en cierto modo como "contradictio que no solo consiste in adjecto sino in nomine" (Abel 319). Las 'palabras primordiales' de Broch (por ejemplo, "calienthelado y sombraluz en dobleorigen" (Broch, Virgilio 102)) sueldan, respectivamente, fusionan los significantes ('caliente' y 'helado' en 'calienthelado') del discurso subjetivo y con esto suturan el estar escindido del sujeto en significantes opuestos (en Lacan: al menos dos significantes, S1 y S2, que constituyen el principio diferencial del lenguaje). A esto le corresponde, dicho sea al pasar, el hecho de que Broch solo considera unidades por encima de esta soldadura, es decir, por sobre el nivel de las palabras, o sea, palabras, oraciones o 'unidades sintácticas' mayores (Broch, $K W 10 / 2$ 150f.). En notoria oposición a Freud ('Signorelli') o James Joyce (Finnegans Wake (cf. Heizmann, "Spätstil” 114)) justamente no disuelve las palabras o sílabas singulares hasta el nivel de los fonemas o morfemas ( $c f$. Lube 44) ${ }^{17}$; el Virgilio de hecho habría sido, según Broch, "escrito en oposición consciente" a Joyce, que para Broch comete un "error lógico": "Si es que se opta por el monólogo interno, entonces nunca debe olvidarse que hay un yo, que el monólogo interno justamente proviene

15 "El 'universo' $[\mathrm{All}]$ para Broch aparentemente no es ni un cosmos muerto ni tan solo la suma de cosas cognoscibles, sino una totalidad psíquicamente vivenciable" (Szabó 264; $c f$. Sidler 79).

16 También Federn enfatizó "el sentimiento de la homogeneidad unitaria de los contenidos yoicos": "el permanente estar presente de una investidura especial y específica, cuya naturaleza consiste en ser una unidad total" (Federn, Ich-Psychologie 210).

17 Una de las pocas excepciones, en la que Broch seguramente se dedica a la descomposición por sonidos de una palabra por debajo de la unidad de la palabra, es la “descomposición fonética de la palabra 'Nada"” (Broch, Lützeler y Federn 179). 
de ese yo [...] y que el yo siempre se percibe como entidad lógica, es decir, como una en la que también lo más contradictorio se desarrolla, alejándose lo uno de lo otro; solamente en los casos clínicos de la desintegración de la personalidad esta línea unitaria se rompe [...]" (Broch y Lützeler, $K W 13,2$ 359).

En semejante soldadura de los significantes, en semejante producción artificial de palabras primordiales autocontradictorias, con Pérennec, puede reconocerse en Broch: "la signification de l'oxymoron: les contradictions fondamentales de l'être humain se fondent à l'approche de la mort en une valeur nouvelle qui transcende leur distinction" (Pérennec 162). Esta soldadura o suturación está basada, por lo visto, en la intención, de parte de Broch, de agotar el discurso del Otro aprovechando al máximo los recursos gramaticales en una especie de lengua fundamental -"hasta la profundidad de su respirar y no-respirar" (Broch, Virgilio 119), como dice en el Virgilio.

No obstante, fácticamente Broch corta las raíces inconscientes del discurso, presuntamente articuladas exhaustivamente, suelda la escisión del sujeto en lugar de ponerlas al descubierto en sus diferencias significantes, tal como ocurre en Joyce o Freud. El remolino metonímico ${ }^{18}$ causado por las oxímora e infinitas parataxis del 'poema sobredimensionado' nunca cesa de no acertar la anhelada 'totalité' o unidad, y temporaliza un "efecto de constante autotraducción" que "no cesa de atravesar sus propias metáforas" (Rabaté 70), respectivamente, no llega detenerse en metáfora absoluta alguna ${ }^{19}$ (acaso así como Lacan construye la metáfora paterna como metáfora absoluta del sujeto). A lo más, el propio Virgilio, in toto, es decir, como gran poema lírico, es, él mismo, tal metáfora. A través de esto -al igual que el texto de la Eneida del protagonista Virgilio en la novela- en cierto modo se convierte en víctima que el autor ofrece a su lector, a saber, para seducirlo, es decir, convertirlo en testigo principal del conjurado Otro absoluto ( $c f$. Dahl 108). Sin embargo, con esto Broch no le hace justicia a la "muerte", que en Virgilio "en cierto modo se convierte en fuerza propulsora de la lengua que oscila 
entre conceptos, metáforas y símbolos" (Szabó 257) y ahí también se expresa en la ornamentación seductora ${ }^{20}$ del estilo literario.

\section{FICCIONES}

"La última frontera era la muerte, pero también esta tenía algo teatral en sí" escribe Hegel sobre la "interioridad abstracta" (Hegel et al. 1076) del mundo romano (su ejemplo es la muerte de Nerón). Esta atmósfera es la misma que respira el Virgilio de Broch. Y la metáfora absoluta (como) la que (se) escenifica el Virgilio de Broch, parece estar acuñada no por último por la propia biografía problemática de su autor: tenía una relación quebrada con sus padres; su único hijo Hermann, al que él llamaba Armand, rompe con él y "a comienzos de los años 30" deja adoptarse por un "padre putativo" (Broch, Broch y Lützeler 17f.), el tío de la madre: Rudolf von Rothermann, de manera que el hijo complementa su nombre paterno convirtiéndolo en " $\mathrm{H}$. F. (Hermann Friedrich) Broch de Rothermann” (Broch, Broch y Lützeler 18). Después de la muerte de Broch, sin embargo, el hijo ingresa a la transmisión de la herencia paterna, a saber, como traductor de Die Verzauberung (Broch, Broch y Lützeler 19f.). Con respecto a la relación padre-hijo es que Broch en el apéndice del Virgilio también se dedica a la relación histórica entre Augusto y Virgilio y cita una leyenda de una traducción al alemán de la Eneida del siglo XVII, según la que Augusto no habría sabido quien era su padre hasta que Virgilio mediante una conjetura lo habría iluminado al respecto.

Con esto, el fingimiento de una metáfora paterna lacaniana, una transmisión subjetivante del nombre-del-padre que recién posibilita la relación con el prójimo, no solamente parece ser "la tarea propiamente tal de la poesía", a saber, bajo la forma de una "verdadera búsqueda del nombre" (Gabbiadini 219), sino también parece haber motivado el que Broch haya emplazado su novela en la antigüedad romana. En la antigüedad romana histórica, la relación padre-hijo es mediada a través de una serie de ficciones jurídicas abstractas y está anudada más fuertemente a "la transmisión de relaciones de dominación" que a meros "problemas legales acerca del patrimonio" (Mayer-Maly 347). El padre, mediante una adopción relativa al derecho de

20 Guilhamon califica los ornamentos estilísticos en el Virgilio de Broch como "seducción y trampa" (241). 
sucesión, puede transferir el patrimonio a cualquier extraño, como consta en el ejemplo paradigmático del Octaviano/Augustus adoptado por Gaius Julius Caesar. Hegel en sus Vorlesungen über die Philosophie der Weltgeschichte en 1826/27 critica "la irrestricta arbitrariedad del testamentar [Testirens] [sic]", que se dirige en "contra del lazo familiar" (Hegel et al. 1075). "A través del principio [abstracto, perteneciente al derecho privado] del testamentar y la forma del matrimonio, los romanos no requerían del amor familiar [...]" (Hegel et al. 1975). El hijo tiene el estatus fingidamente-legal de un esclavo que solo puede volverse libre y autónomo (emancipatio) en un juego amañado, a saber, al ser vendido tres veces a un mediador ${ }^{21}$. E incluso entonces el hijo aún no nacido (nasciturus) en la muerte del padre está sujeto a una relación objetal. Cuando Hegel identifica a "Cristo" como el nasciturus divino, como unidad de la moral [Sittlichkeit] familiar y del "lazo" social (Hegel et al. 1077), también el autor del Virgilio parece hegeliano, a saber, como alguien que quiere reemplazar ficciones jurídicas por ficciones religiosas o bien literarias. Y así Broch en 1934 escribe en una carta, por cierto, aún antes del Virgilio: "Usted [Egon Vietta] tiene toda la razón cuando constata que no logro librarme de Hegel; considero que mis intentos histórico-filosóficos, por mucho que en cuanto a su contenido se alejen de Hegel, metodológicamente son del todo hegelianos" (Broch y Lützeler, $K W$ 13,1 277).

Desde un punto de vista moderno, para semejantes ficciones probablemente valga lo mismo que para las jurídicas: la "apariencia de la continuidad" que ellas producen es empleada la mayoría de las veces justamente "donde el mismo legislador está en duda" (Larenz 252). Y también según Freud, a propósito del hombre moderno, "[e]s imposible moverlo a que renuncie, nada menos que en el tratamiento de sus intereses más importantes, a las certezas que suele pedir en todas sus actividades habituales. Me acuerdo de uno de mis hijos que se distinguió desde muy temprano por una particular insistencia en lo fáctico, positivo. Toda vez que se relataba a los niños un cuento que escuchaban con recogimiento, él venía y preguntaba: «¿Es una historia verdadera?». Habiéndosele respondido que no, se alejaba con ademán de menosprecio. Es de esperar que pronto los seres humanos adopten parecido comportamiento frente a los cuentos religiosos, a despecho de la recomendación del «como si»" (Freud, "Zukunft” 351). El autor Broch ante 
esta pregunta parece ambivalente: por un lado, “[...] soy de la opinión de que todo lo religioso, todo lo místico y, con esto, finalmente lo "verdadero en sí' siempre es un enunciado acerca de la estructura de la conciencia [...]" (Broch y Lützeler, $K W 13,2$ 440), así Broch en una carta de 1945 a Aldous Huxley, y que la introspección de Virgilio justamente es "[...] que al arte y solo al arte le es concedido el don de dejar intuir lo aún-impronunciable y, sin embargo, ya presente" (Broch y Lützeler, $K W$ 13, 2 455). Por el otro lado, considera que el "poeta" está "obligado a forjarse una opinión ética y metafísica" (Broch y Lützeler $K W 13,2$ 360) y esta en su "psicología de las masas" concierne "en última instancia a la misma problemática que en el Virgilio", a saber, "los procesos [...] que conducen a los hombres a la pérdida y recuperación de sus vérités fondamentales, es decir, sus actitudes religiosas" (Broch y Lützeler, $K W 13,2$ 454). En el fondo se trata del "despertar" (Broch y Lützeler, KW 13,2 455), así Broch, es decir, de tales "verdades fundamentales' "y de su refundamentación en el alma humana" (Broch y Lützeler, $K W$ 13, 2 456). El que la ficción literaria de Broch solo constituye la forma de tal despertar y no también a su contenido, el que más bien es adoptado de la tradición religiosa, aparece como déficit central de la novela Virgilio. No ofrece una "ganancia en cuanto a su realidad", por muy errática que fuera, en el sentido de Federn (Ich-Psychologie 148), es decir, un acceso a la realidad psíquica inconsciente de los contemporáneos de Broch. El mismo Broch "despertó", experimentó su propio despertar en esto hay que darle la razón a Hannah Arendt-, "cuando comenzó la noche”, "en la última fase del oscurecimiento en Europa": "Despertó en una realidad que lo superó hasta tal punto que de inmediato la tradujo en un sueño, tal como lo hace un hombre que se levanta desde la noche. Este sueño es 'La muerte de Virgilio" (Arendt 171). Si bien, entonces, el Virgilio puede ser interpretado como una especie de sueño en el sueño, no obstante, su forma literaria sigue siendo una "forma de rechazo" (Freud, Traumdeutung 344) de esta realidad, tal como ocurre en el caso del habitual "sueño en el sueño" (343) según la Traumdeutung freudiana; una forma de rechazo que no llega hasta el fondo de esta realidad y que, por ende, no puede lidiar con ella. "La intención es también desvalorizar a lo «soñado» del sueño, arrebatarle su realidad; lo que se sigue soñando después de despertar del «sueño dentro del sueño» es lo que el deseo onírico quiere poner en lugar de la realidad borrada. Puede entonces suponerse que lo «soñado» contiene la figuración de la realidad, el recuerdo real, y el sueño que sigue, al contrario, la figuración de lo que el soñante meramente desea" (343). 


\section{BIBLIOGRAFÍA}

Abel, Carl. "Über den Gegensinn der Urworte". Sprachwissenschaftliche Abhandlungen. Leipzig: Verlag von Wilhelm Friedrich, 1885. 311-68.

Arendt, Hannah. "Nicht mehr und noch nicht: Hermann Brochs 'Der Tod des Vergil' (1946)". Briefwechsel 1946 Bis 1951. Hannah Arendt; Hermann Broch. Ed. Lützeler, Paul Michael. Frankfurt/M.: Jüdischer Verlag, 1996. 169-74.

Arieti, Silvano. Interpretation of Schizophrenia. 2a . ed. 1955. New York: Basic Books, 1974. Brinkmann, Richard. “On Broch's Concept of Symbol”. Hermann Broch: Literature, Philosophy, Politics. Ed. Stephen D. Dowden. Columbia, SC: Camden House. 193-206, 1988.

Broch, Hermann. Der Tod des Vergil. Frankfurt/M.: Suhrkamp, 1995.

Kommentierte Werkausgabe Band 10/2: Wert- und Geschichtstheorie; Erkenntnistheorie. Frankfurt/M.: Suhrkamp, 1977.

"Werttheoretische Anmerkungen zur Psychoanalyse (1936)". Kommentierte Werkausgabe Band 10/2: Philosophische Schriften 2 Theorie. 2. Aufl. Frankfurt/Main: Suhrkamp, 1986. 173-94.

Broch, Hermann, Armand Broch, and Paul Michael Lützeler. Verlorener Sohn? Hermann Brochs Briefwechsel mit Armand; 1925-1928. 1. Aufl. Frankfurt am Main: Suhrkamp, 2010.

Broch, Hermann y Paul Michael Lützeler. Briefe (1913 - 1938). Kommentierte Werkausgabe: Briefe 1 (1913 - 1938). 1. Aufl. Frankfurt am Main: Suhrkamp, 1981.

Briefe (1938 - 1945). Kommentierte Werkausgabe: Briefe 2 (1938 - 1945). 1. Aufl. Frankfurt am Main: Suhrkamp, 1981.

Massenwahntheorie. Beiträge zu Einer Psychologie der Politik. Kommentierte Werkausgabe; Bd. 12. 1. Aufl. Frankfurt am Main: Suhrkamp, 1979.

Psychische Selbstbiographie. 1. Aufl. Frankfurt am Main: Suhrkamp, 1999.

Broch, Hermann, Paul Michael Lützeler y Paul Federn. "Frauengeschichten". Briefe an Paul Federn; 1939-1949. 1. Aufl. Frankfurt am Main: Suhrkamp, 2007.

Dahl, Gerhard. "Hermann Broch: 'Der Tod Des Vergil'. Eine Psychoanalytische Studie". Psychoanalytische Textinterpretation. Ed. Cremerius, Johannes. Hamburg: Hoffmann und Campe, 1974. 71-128.

Edelmann, Thomas, ed. "Literaturtherapie wider Willen”. Hermann Brochs Traum-Dichtung Zwischen Metaphysik und Psychoanalyse. Würzburg: Königshausen und Neumann, 1997.

Federn, Paul. Ich-Psychologie und die Psychosen. Bern: Hans Huber, 1956.

Zur Psychologie Der Revolution: Die Vaterlose Gesellschaft. Leipzig, Wien: Anzengruber-Verlag, 1919.

Freud, Sigmund. Das Unbehagen in der Kultur. Wien: Internationaler Psychoanalytischer Verlag, 1930.

"Die Traumdeutung”. Über Den Traum. Gesammelte Werke II/III. Frankfurt/M.:

Fischer Taschenbuch Verlag, 1999.

Die Zukunft Einer Illusion. Gesammelte Werke Band XIV. Frankfurt/M.: Fischer Taschenbuch Verlag, 1999. 323-80. 
Gabbiadini, Guglielmo. Die 'Weltanschauung'Des Dichters in Hermann Brochs Der Tod Des Vergil. Hermann Brochs Vergil-Roman: Literarischer Kontext und kulturelle Konstellationen. Ed. Agazzi, Elena. Stauffenburg: Tübingen, 2016. 213-38.

Guattari, Félix. Psychotherapie, Politik und die Aufgaben der institutionellen Analyse. 1. Aufl. Frankfurt am Main: Suhrkamp, 1976.

Guilhamon, Elizabeth. Hermann Broch, Der Tod des Vergil: Ornament und Enthüllung - Das letzte Paradoxon. Hermann Brochs Vergil-Roman: Literarischer Intertext und kulturelle Konstellation. Ed. Elena Agazzi et al. Tübingen: Stauffenburg, 2016. 239-51.

Hegel, G.W.F. Phänomenologie des Geistes. Leipzig: Verlag der Dürr'schen Buchhandlung, 1907. Hegel, Georg Wilhelm Friedrich et al. "Nachschriften zu dem Kolleg des Wintersemesters 1826/27”. Vorlesungen Zur Philosophie der Weltgeschichte. Gesammelte Werke. Hamburg: Felix Meiner Verlag, 2019.

Heizmann, Jürgen. Antike und Moderne in Hermann Brochs 'Tod des Vergil'. Über Dichtung und Wissenschaft, Utopie und Ideologie. Tübingen: Narr, 1997.

"Spätstil. Hermann Brochs der Tod des Vergil und James Joyces Finnegans Wake". Hermann Brochs Vergil-Roman: Literarischer Intertext und kulturelle Konstellationen. Ed. Elena Agazzi. Tübingen: Stauffenburg, 2016. 107-22.

Illi, Manuel. "Signal und Sigel. Hermann Brochs Sprach- und Symboltheoretische Reflexionen Zwischen Romantik und Neopositivismus". Hermann Broch und die Romantik. Ed. Doren Wohlleben et al. Berlin: De Gruyter, 2014. 87-106.

Jenkins, Jennifer. "Broch's der Tod des Vergil: Art and Power, Language and the Ineffable". A Companion to the Works of Hermann Broch. Ed. Bartram, Graham. Rochester: Camden, 2019. 189-206.

Jung, Carl Gustav. “Zur Gegenwärtigen Lage der Psychotherapie”. Zentralblatt für Psychotherapie und ihre Grenzgebiete einschliesslich der medizinischen Psychologie und psychischen Hygiene 7 (1934).

Lacan, Jacques. Le Séminaire Livre III: Les Psychoses. Paris: Éditions du Seuil, 1981. Le Séminaire Livre XIII: L'objet de la Psychanalyse. Paris: AFI, 1964-65. Le Séminaire Livre XV: L'acte Psychanalytique. Bregenz: Lacan-Archiv, 1967-68. Le Séminaire Livre XVI: D’un Autre à l'autre. Bregenz: Lacan-Archiv, 1968-69. Le Séminaire Livre XXIII: Le Sinthome. Bregenz: Lacan-Archiv, 1975-76.

Larenz, Karl. Methodenlehre der Rechtswissenschaft. Enzyklopädie der Rechts- und Staatswissenschaft: Abteilung Rechtswissenschaft 5, neu bearb. Aufl. Berlin [u.a.]: Springer, 1983.

Lawrence, Joseph P. "Broch's Translinguist Poetics: A Response to Kuno Lorenz”. Hermann Broch. Literature, Philosophy, Politics. Ed. Dowden, Stephen. Columbia, SC: Camden House, 1988. 305-322.

Lévy-Bruhl, Lucien. Les Fonctions Mentales dans les Sociétés Inférieures. Paris: Alcan, 1910. Lube, Barbara. Sprache und Metaphorik in Hermann Brochs Roman "Der Tod Des Vergil". Frankfurt/Main: Lang, 1986.

Lützeler, Paul Michael. Hermann Broch und die Moderne. Roman, Menschenrecht, Biografie. Paderborn [u.a.]: Fink, 2011. 
ed. Hermann Broch. Eine Biographie. 1. Aufl. Frankfurt am Main: Suhrkamp, 1988.

Martens, G. "Hermann Broch and the dilemma of literature in the modern age". In G. Bartram (Hg.). A Companion to the Works of Hermann Broch. Rochester: Camden, 2019. 59-72.

Mayer-Maly, Theo. "Erbrecht”. Der Kleine Pauly. Lexikon der Antike. München: Deutscher Taschenbuch Verlag, 1979. 346-49. Vol. 2.

Mondon, Christine. "Hermann Broch und die Psychoanalyse". Hermann Broch: Neue Studien. Ed. Michael Kessler. Tübingen: Stauffenburg, 2003. 510-23.

Pérennec, Marie-Hélène. “'Der Tod des Vergil': Délire Verbal ou Création Langagière? Le Point de Vue d'un Grammairien". Broch. Ed. Jean-Charles Margotton. Vol. 16. Cahiers d'études Germaniques. Lyon/Nice/Marseille/Montpellier 1989.

Popper, Karl. Logik der Forschung. 11. Aufl. Tübingen: Mohr Siebeck, 2005.

Rabaté, Jean-Michel. "Broch, Traduit”. Broch. Ed. Margotton, Jean-Charles. Vol. 16. Cahiers d'études germaniques. Lyon/Nice/Marseille/Montpellier 1989. 59-70.

Ritzer, Monika. "Experimente mit der Psyche: Hermann Broch und C. G. Jung”. Hermann Broch: Neue Studien. Ed. Michael Kessler. Tübingen: Stauffenburg, 2003. 524-52.

Roesler-Graichen, Michael. Poetik und Erkenntnistheorie. Hermann Brochs "Tod des Vergil” zwischen logischem Kalkül und phänomenologischem Experiment. Würzburg: Königshausen und Neumann, 1994.

Roethke, Gisela. Zur Symbolik in Hermann Brochs Werken. Platons Höhlengleichnis als Subtext. Edition Orpheus; 4. Tübingen: Francke, 1992.

Sidler, Judith. Literarisierter Tagtraum. Einheitskonstruktionen in Hermann Brochs "TierkreisErzählungen”. Königshausen \& Neumann, 2003.

Szabó, László V. "Das Vexierspiel der Begrifflichkeit und Metaphorik in Hermann Brochs Der Tod Des Vergil". Hermann Brochs Vergil-Roman: Literarischer Intertext und kulturelle Konstellation. Ed. Elena Agazzi et al. Tübingen: Stauffenburg, 2016. 253-68.

Vergilius Maro, Publius; Niklas Holzberg and Markus Schauer, eds. Aeneis. LateinischDeutsch. Berlin: De Gruyter, 2015.

von Domarus, Eilhard. "Beispiele Paralogischen Denkens in der Schizophrenie". Zeitschrift für die gesamte Neurologie und Psychiatrie 90 (1) (1924). 620-27.

The Logical Structure of Mind. An inquiry into the philosophical foundation of psychology and psychiatry. Web $<\mathrm{https}$ ://core.ac.uk/download/pdf/85255015.pdf $>$.

"Prälogisches Denken in der Schizophrenie". Zeitschrift für die gesamte Neurologie und Psychiatrie 87 (1) (1923). 84-93.

"The Specific Laws of Logic in Schizophrenia". Language and Thought in Schizophrenia. Ed. Kasanin, J.S. New York: Norton \& Company, 1964 (1944). 104-14.

"Zur Theorie des Schizophrenen Denkens". Zeitschrift für die gesamte Neurologie und Psychiatrie 108 (1) (1927). 703-14.

Waldeck, Peter Bruce. Die Kindheitsproblematik bei Hermann Broch. München-Allach: Fink, 1968.

Weiss, Walter. “"Mathematisches Mysterium': Mysticism and Metaphor in the Writing of Hermann Broch". Hermann Broch. Literature, Philosophy, Politics. The Yale Symposium 1986. Ed. Stephen D. Dowden, Vol. 30. Columbia, SC: Camden House, 1988. 